\title{
Advanced Computer Network for Healthcare Sector - Liver Function Diagnosis
}

\author{
Kedri Janardhana ${ }^{1}$, P. Rajasekar ${ }^{2}$, A. Shali ${ }^{3}$, M. Vijayaragavan ${ }^{4}$, D. Stalin David ${ }^{5}$ \\ \{janardhankedri@dei.ac.in ${ }^{1}$, rajasekp@srmist.edu.in², shali.cse@sairam.edu.in ${ }^{3}$, \\ mrvijay.ragavan@gmail.com ${ }^{4}$, sdstalindavid707@gmail.com 5 \\ ${ }^{1}$ Assistant Professor (Senior Grade), Department of Electrical Engineering, Faculty of Engineering, \\ Dayalbagh Educational Institute (Deemed to be University), Agra, India, ${ }^{2}$ Assistant Professor, \\ Department of Information Technology, S.R.M Institute of Science and Technology, ${ }^{3}$ Assistant Professor, \\ Department of Computer Science and Engineering, Sri Sairam Engineering College, Chennai, \\ India, ${ }^{4}$ Assistant Professor, Department of Electrical and Electronics Engineering, Mailam Engineering \\ College, Mailam, India, ${ }^{5}$ Assistant Professor, Department of Computer Science and Engineering, IFET \\ College of Engineering, Villupuram, India
}

\begin{abstract}
Innovation technology has been implemented in different field, especially in life science, for example, genome sequencing, hereditary printing, proteomic and metabolomic examination, electronic clinical records, and patient-revealed wellbeing information have delivered a wide scope of information, from an assortment of people, cell types, and problems (huge information). Notwithstanding, this information should be incorporated and dissected in the event that it is to deliver models or ideas about physiological working or pathogenesis components. The majority of this information is freely accessible, which permits specialists anyplace to look for side effects of explicit biologic cycles or helpful targets for explicit infections or sorts of patients. We are evaluating ongoing advances in the field of computational and foundational science, and featuring the prospects of analysts utilizing enormous informational collections in the fields of gastroenterology and hepatology, to supplement conventional techniques for demonstrative and restorative access. We present and look at two AI calculations, which naturally create choice trees from research facility information. TheBayesNet classifier gives that the highest accuracy level which is $82.68 \%$, and the highest precision value is $79.94 \%$ which is produced by NaivebayesMultinomial algorithm. This system recommends that the BayesNet classifier and NaivebayesMultinomial approaches. It produce the optimal result compare with other classifiers.
\end{abstract}

Keywords: Classifier, Big data, Precision, Accuracy, algorithm, Recall.

\section{Introduction}

These days world appears to base upon PCs in each part of regular day to day existence. Medical services is one of the areas where computerization by executing IT arrangements is important to work with crafted by its associations. The enormous number of records containing the wellbeing data of many patients requests an efficient construction and probability of electronic information trade to improve the data the executives and in this manner the foundation the board. It is crucial for structure a framework to orchestrate and incorporating guidelines that will meet clinical and business needs for dividing data between associations and frameworks. This would mean carrying out enormous scope incorporated IT 
arrangements in medical services. The board of an essential medical services establishment now and again can be alluded to as the administration of little/medium endeavor (SME). This is an exceptionally significant issue because of the reality, that SMEs are right now driving the entire european economy. The point of this paper is to momentarily introduce the possibility of an organization for an essential medical services foundation, including numerous useful highlights making the work in this sort of an establishment simpler and more viable, empowering better data the executives.

In this portion uncovers the presence of this investigation of examination work. Logical pathways rely upon ace standards ("expecting... by then... else"), which can be imagined as the choice trees [1-5]. Utilizing input data from a conveyed report on hepatitis $\mathrm{C}$ patients, we show that the two calculations are definitely not hard to apply and make possible choice trees [6-8]. These calculations attest normal data about the power of examination office testing to distinguish liver fibrosis and cirrhosis. In clinical practice, characteristic pathways address "savvy test profiles", which are followed just to where an illustrative decision can be made Machine learning estimations may be used to either favor the choice trees set up by human trained professionals or to suggest anticipated new trees, if rules are not available[9-16].

In this paper, we present and survey "party unit", a quantifiable programming apparatus [17-22], which normally makes decision trees from veritable exploration office data. But such PC driven approaches have been pursued for some time[23-26], applications for lab diagnostics have been sparse so far[27].In this paper presents region 2 of this paper explains the detail on the associated works. In portion 3 presents the materials and strategies embraced and territory 4 presents the nuances of the examinations and discussions. Finally section 5 wraps up the paper by sharing our derivations and likely game plans.

\section{Materials And Methods}

In this section presents the materials and methods of this research work. The dataset collected from UCI repository. The data set contains laboratory values of blood donors and Hepatitis $\mathrm{C}$ patients and demographic values like age. The below information have given about the list of the attributes.

\begin{tabular}{|l|l|}
\hline S.No & Attribute \\
\hline 1 & X (Patient ID/No.) \\
\hline 2 & $\begin{array}{l}\text { Category } \quad(\text { O=Blood Donor, 0s=suspect Blood Donor, 1=Hepatitis, } \\
\text { =Fibrosis, 3=Cirrhosis) }\end{array}$ \\
\hline 3 & Age \\
\hline 4 & Sex (female/male) \\
\hline 5 & Alanine transaminase (ALT) \\
\hline 6 & Aspartate aminotransferase (AST) \\
\hline 7 & Alkaline phosphatase (ALP) \\
\hline 8 & Albumin \\
\hline 9 & Bilirubin \\
\hline
\end{tabular}




\begin{tabular}{|l|l|}
\hline 10 & alpha-fetoprotein \\
\hline 11 & agglutinin-reactive AFP \\
\hline 12 & PIVKA-II \\
\hline 13 & 8-glutamyltranspedtidase \\
\hline
\end{tabular}

The Weka 3.8.9 has implemented to get the optimal solution of the above dataset. The below approaches have implemented and got optimal solution.

- BayesNet:Bayes

- NaiveBayes:

- NaiveBayesMultinomial:

- NaiveBayesMultinomialText:

- NaiveBayesMultinomialUpdateable:

- NaiveBayesUpdateable:

\section{Results And Discussions}

In this section focuses the results and discussions of this research work. The below table clearly demonstrates that the Accuracy levels of all approaches namely BayesNet, NaiveBayes, NaiveBayesMultinomial, NaiveBayesMultinomialText, NaiveBayesMultinomialUpdateableandNaiveBayesUpdateabl. All of these algorithms belong to Bayes classifier.

Table 1: List of Bayes Classifiers with their metrics

\begin{tabular}{|l|l|l|}
\hline S.No & Algorithm & Accuracy \\
\hline 1 & BayesNet & $75.86 \%$ \\
\hline 2 & NaiveBayes & $72.51 \%$ \\
\hline 3 & NaiveBayesMultinomial & $65.19 \%$ \\
\hline 4 & NaiveBayesMultinomialText & $81.43 \%$ \\
\hline 5 & NaiveBayesMultinomialUpdateable & $78.69 \%$ \\
\hline
\end{tabular}




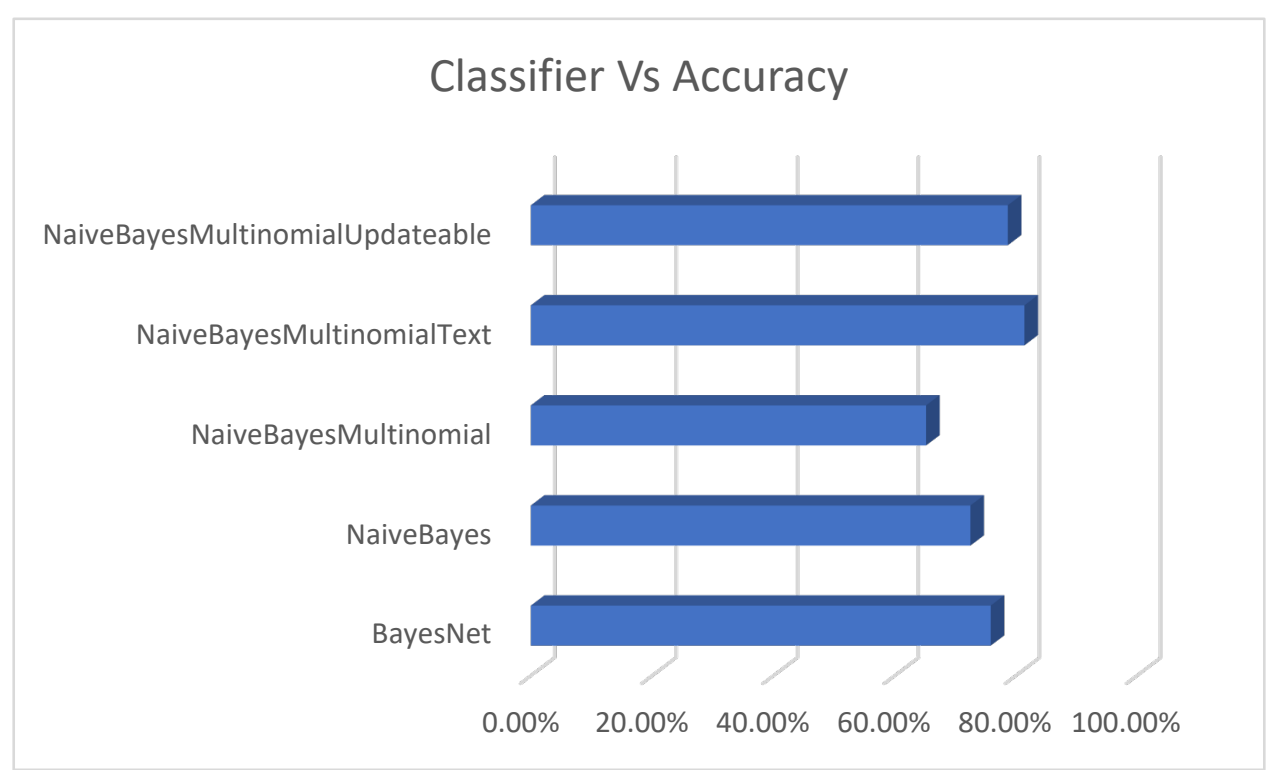

\section{Figure 1: Classifier VsAlgorithm Accuracy}

This above diagram clearly represents that the BayesNet produces $75.86 \%$ of accuracy level , NaiveBayes holds $72.51 \%$ accuracy level, NaiveBayesMultinomial is holding $65.19 \%$ accuracy level, NaivBayesMultinomialText gives the accuracy level is $81.43 \%$, NaiveBayesMultinomialUpdateable gives that $78.69 \%$ accuracy level. All the leading Bayes classifiers have produce the accuracy level is $65 \%$ and the highest accuracy level is $81.43 \%$.

NaiveBayesMultinomial, NaivBayesMultinomialText, NaiveBayesMultinomialUpdateable and NaiveBayes have hold above $72 \%$ to $77 \%$ of accuracy level. The BayesNet classifier gives that the highest accuracy level which is $81.43 \%$.

Table 2: List of Bayes Classifiers with Precision Value

\begin{tabular}{|l|l|l|}
\hline S.No & Algorithm & Precision \\
\hline 1 & BayesNet & $79.14 \%$ \\
\hline 2 & NaiveBayes & $79.23 \%$ \\
\hline 3 & NaiveBayesMultinomial & $79.94 \%$ \\
\hline 4 & NaiveBayesMultinomialText & $79.57 \%$ \\
\hline 5 & NaiveBayesMultinomialUpdateable & $74.67 \%$ \\
\hline
\end{tabular}

The above table clearly demonstrates that the precision call values of various approaches namely BayesNet, NaiveBayes, NaiveBayesMultinomial, NaiveBayesMultinomialText, NaiveBayesMultinomialUpdateableandNaiveBayesUpdateabl. All of these algorithms belong to Bayes classifier. 


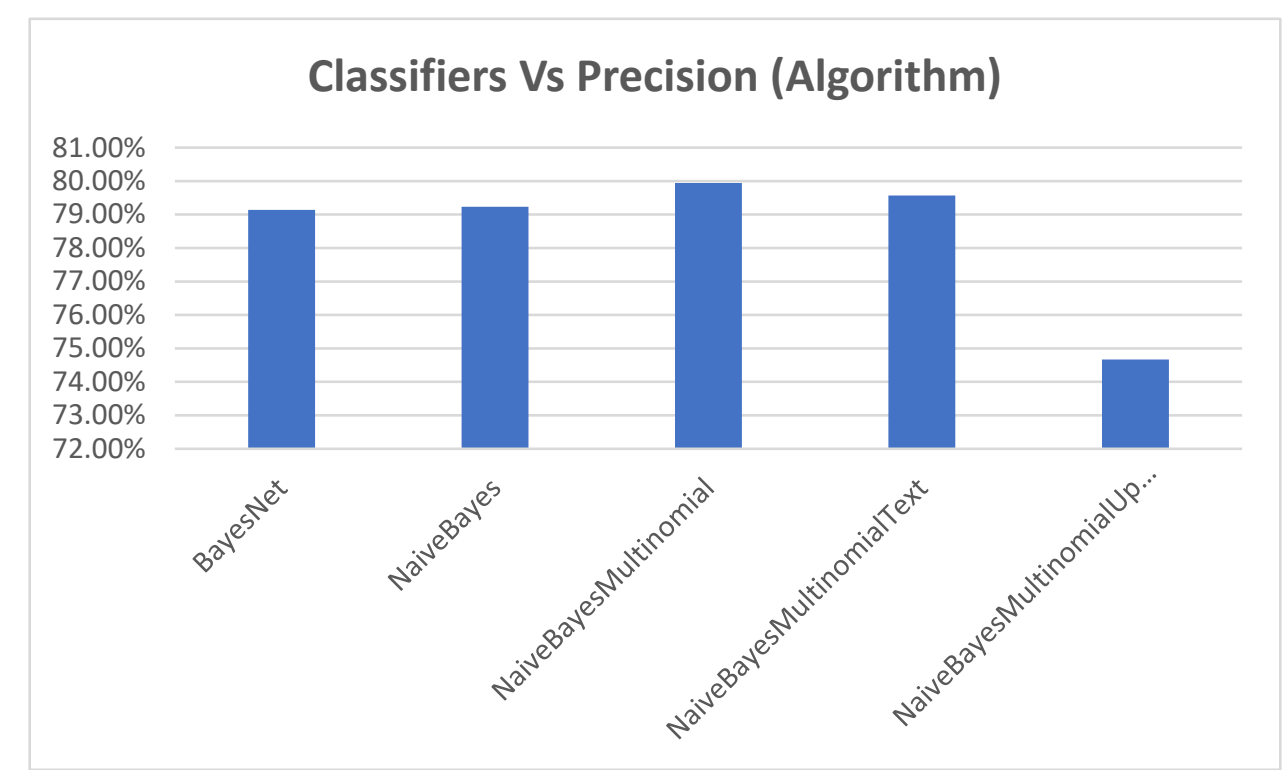

Figure 2: Classifiers Vs Precision (Algorithm)

The above diagram represents that the BayesNet produces $79.14 \%$ of precision value, NaiveBayes holds $79.23 \%$ precision value, NaiveBayesMultinomial is holding $79.94 \%$ precision value, NaivBayesMultinomialText gives the precision value is $79.57 \%$, NaiveBayesMultinomialUpdateable gives that $74.67 \%$ precision value and NaiveBayes has $74.54 \%$ of precision value. All the leading Bayes classifiers have produce the precision value is above $74 \%$ and the highest precision value is $79.94 \%$ which is produced by NaivebayesMultinomial algorithm.BayesNet, NaiveBayesMultinomial, NaivBayesMultinomialText, NaiveBayesMultinomialUpdateable and NaiveBayes have hold the precision value from $74 \%$ to $80 \%$.

The below table clearly represents that the recall call values of various approaches namely BayesNet, NaiveBayes, NaiveBayesMultinomial, NaiveBayesMultinomialText,NaiveBayesMultinomialUpdateableand NaiveBayesUpdateabl. All are belonging to Bayes classifier.

Table 3: Various Approaches Vs Recall Values

\begin{tabular}{|l|l|l|}
\hline S.No & Algorithm & Recall \\
\hline 1 & BayesNet & $83.18 \%$ \\
\hline 2 & NaiveBayes & $70.56 \%$ \\
\hline 3 & NaiveBayesMultinomial & $71.57 \%$ \\
\hline 4 & NaiveBayesMultinomialText & $73.76 \%$ \\
\hline 5 & NaiveBayesMultinomialUpdateable & $75.45 \%$ \\
\hline
\end{tabular}




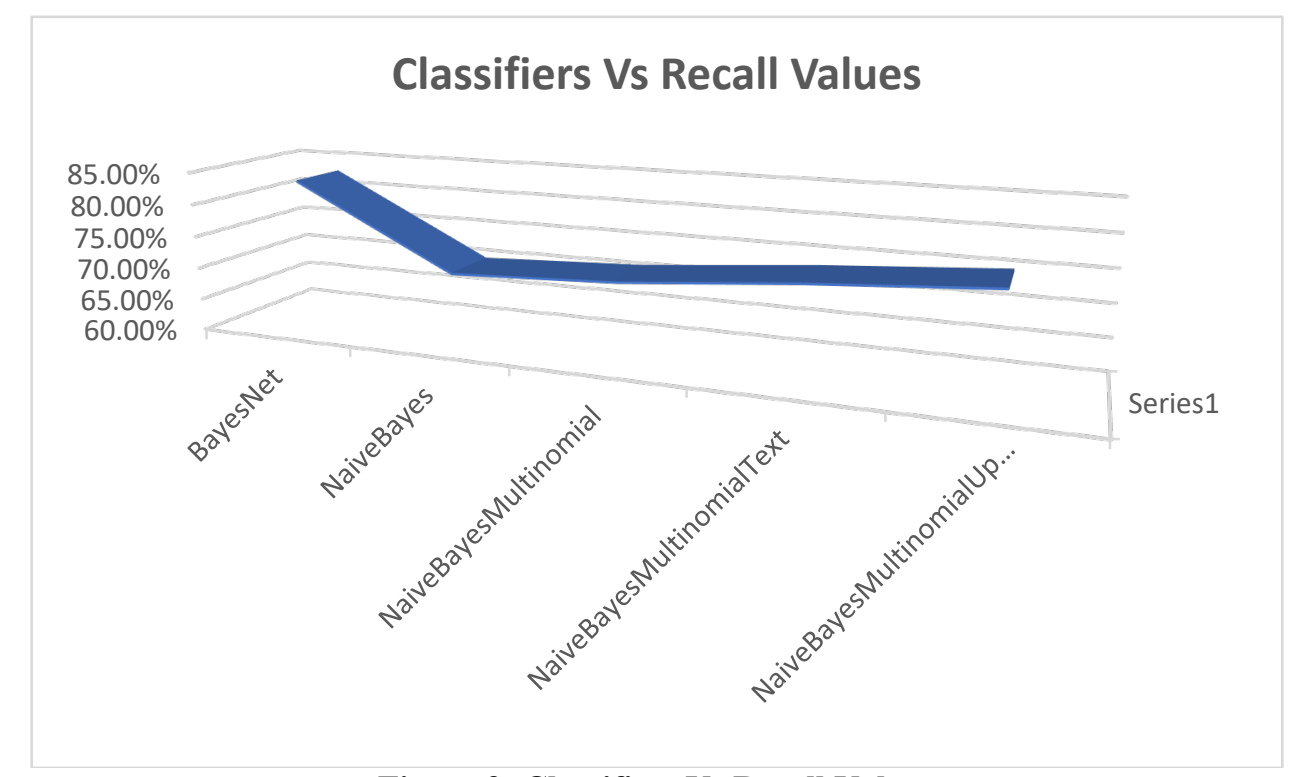

Figure 3: Classifiers Vs Recall Values

The above graph addresses that review esteems have been delivered by utilizing different calculation the BayesNet produces $83.18 \%$ of Recall esteem, NaiveBayes holds $70.56 \% \%$ of review esteem, NaiveBayesMultinomial is holding $71.57 \%$ of review esteem, NaivBayesMultinomialText gives the review esteem is $73.76 \%$, NaiveBayesMultinomialUpdateable gives that $75.45 \%$ review esteem and NaiveBayesUpdateable has $75.98 \%$ of review esteem. All the main Bayes classifiers have produce the review esteem is above $70 \%$ and the most elevated review esteem is $83.18 \%$ which is delivered by BayesNet calculation. BayesNet, NaiveBayesMultinomial, NaivBayesMultinomialText, NaiveBayesMultinomialUpdateable and NaiveBayes have hold the review esteem from $70 \%$ to $84 \%$.

The above chart addresses that all methodologies have set aside the effort to construct the model like BayesNet sets aside the effort to assemble the model around 0.18 seconds, NaiveBayes requires 0.2 seconds to fabricate the model, NaiveBayesMultinomial sets aside the effort to fabricate the model around 0.48 seconds, NaiveBayesMultinomialText requires 0.24 seconds to fabricate the model, NaiveBayesMultinomialUpdateable methodology requires 0.22 seconds to fabricate the model and NaiveBayesUpdateable requires 0.9 seconds to fabricate the model.

The BayesNet is taking low time utilization to fabricate the model. It requires just 0.18 seconds. It is low time utilization contrast and different methodologies for building the models. NaiveBayes and NaiveBayesMultinomialUpdateable have same time utilization to assemble the model. It require 0.20 seconds to construct the model. NaiveBayesMultinomialText is requiring 0.24 seconds to construct the model, NaiveBayesMultinomial requires 0.48 seconds to fabricate the model lastly, NaivebayesUpdateable requires 0.9 seconds to assemble the new model. This work unmistakably shows that the NaiveBayesUpdateable sets aside more effort to fabricate the model i.e., 0.9 seconds and BayesNet takes less time utilization to assemble the model. The NaiveBayes ,NaiveBayesMultinomialUpdateabl and NiaveBayesMultinomialText are taking 
0.2 to 0.24 seconds. The remainder of the methodologies are requiring more than 0.48 seconds.

\section{Conclusion}

At long last this work finishes up, The BayesNet classifier gives that the most elevated exactness level which is $82.68 \%$, NaiveBayesMultinomial, NaivBayesMultinomialText, NaiveBayesMultinomialUpdateable and NaiveBayes have hold above $72 \%$ to $77 \%$ of precision level. . All the main Bayes classifiers have produce the exactness esteem is above $74 \%$ and the most noteworthy accuracy esteem is $79.94 \%$ which is created by NaivebayesMultinomial calculation. This framework suggests that the BayesNet classifier and NaivebayesMultinomial approaches. It produce the ideal outcome contrast and other classifiers.the most noteworthy review esteem is $83.18 \%$ which is created by BayesNet calculation and rest of the BayesNet, NaiveBayesMultinomial, NaivBayesMultinomialText, NaiveBayesMultinomialUpdateable and NaiveBayes have hold the review esteem from $70 \%$ to $84 \%$. The BayesNet is taking low time utilization to construct the model. It requires just 0.18 seconds. It is exceptionally low time utilization contrast and different methodologies for building the models.

Albeit numerous standard parts of medical services don't need advance organizations, there are exceptional issues in a scope of medical services claims to fame that require their utilization. Progressed network applications can straightforwardly and in a roundabout way influence wellbeing results. They improve ways the medical services local area can share assets and cooperate with patients, understudies, and each other by giving approaches to appropriate data, team up, and work in a more versatile climate. They additionally present difficulties to engineers, end clients, and organization chairmen in light of the fact that they regularly change how organizations are overseen and medical services is given. Progressed networks are not static, nor are the requirements of medical care.

\section{References}

[1] Dua, D. and Graff, C. (2019). UCI Machine Learning Repository [http://archive.ics.uci.edu/ml]. Irvine, CA: University of California, School of Information and Computer Science.

[2] Hoffmann G, Aufenanger J, Foedinger M, et al. Benefits and limitations of laboratory diagnostic pathways. Diagnosis 2014;1:269-76.

[3] Hofmann W, Aufenanger J, Hoffmann G, et al. editors.Laboratory diagnostic pathways. Berlin: De Gruyter, 2016.

[4] Thomas L, Thomas C. Detection of iron restrictioninanaemic and non-anaemic patients: New diagnostic approaches. Eur J Haematol 2017;99:262-8.

[5] Hothorn T, Zeileis A. partykit: A modular toolkit for recursive partytioning in R. J Machine Learning Res 2015;16:3905-9.

[6] Murthy S. Automatic construction of decision trees from data: a multi-disciplinary survey. Data Mining and Knowledge Discovery 1998;2:345-89.

[7] Qu Y, Adam BL, Yasui Y, et al. Boosted decision tree analysis of SELDI mass spectral serum profiles discriminates prostate cancer from noncancer patients. ClinChem 2002;48:1835-43.

[8] Dórea FC, Muckle CA, Kelton D, et al. Exploratory analysis of methods for automated classification of laboratory test orders into syndromic groups in veterinary medicine. PLoS One 2013;8:e57334. 
[9] Metthin E, Veen J, Dekhuijzen R, et al. Development of a diagnostic decision tree for obstructive pulmonary diseases base on real-life data. ERJ Open Res 2016;2.

[10] Lichtinghagen R, Pietsch D, Bantel H, et al. The Enhanced Liver Fibrosis (ELF) score: Normal values, influence factors and proposed cut-off values. J Hepatol 2013;59:236-42.

[11] Khan NA, Quan H, Bugar JM, Lemaire JB, Brant R, Ghali WA. Association of postoperative complications with hospital costs and length of stay in a tertiary care center. J Gen Intern Med. 2006;21(2):177-80. Epub 2006/04/12. JGI319 [pii] pmid:16606377

[12] Hobson C, Ozrazgat-Baslanti T, Kuxhausen A, Thottakkara P, Efron PA, Moore FA, et al. Cost and Mortality Associated With Postoperative Acute Kidney Injury. Ann Surg. 2014. Epub 2014/06/03

[13] Korenkevych D, Ozrazgat-Baslanti T, Thottakkara P, Hobson CE, Pardalos P, Momcilovic P, et al. The Pattern of Longitudinal Change in Serum Creatinine and 90-Day Mortality After Major Surgery. Ann Surg. 2015.

[14] Bihorac A, Brennan M, Ozrazgat-Baslanti T, Bozorgmehri S, Efron PA, Moore FA, et al. National surgical quality improvement program underestimates the risk associated with mild and moderate postoperative acute kidney injury. Crit Care Med. 2013;41(11):2570-83. pmid:23928835

[15] Bihorac A, Yavas S, Subbiah S, Hobson CE, Schold JD, Gabrielli A, et al. Long-term risk of mortality and acute kidney injury during hospitalization after major surgery. Ann Surg. 2009;249(5):851-8. pmid:19387314

[16] Huber M, Ozrazgat-Baslanti T, Thottakkara P, Efron PA, Feezor R, Hobson C, et al. Mortality and Cost of Acute and Chronic Kidney Disease after Vascular Surgery. Annals of vascular surgery. 2015.

[17] Ozrazgat-Baslanti T, Thottakkara P, Huber M, Berg K, Gravenstein N, Tighe P, et al. Acute and chronic kidney disease and cardiovascular morltaity after major surgeyr Annals of Surgery. 2015.

[18] Zarjou A, Agarwal A. Sepsis and acute kidney injury. J Am SocNephrol. 2011;22(6):9991006. Epub 2011/05/14. ASN.2010050484 [pii]. pmid:21566052

[19] Copeland GP, Jones D, Walters M. POSSUM: a scoring system for surgical audit. Br J Surg. 1991;78(3):355-60. Epub 1991/03/01. pmid:2021856

[20] Lake AP, Williams EG. ASA classification and perioperative variables: graded anaesthesia score? Br J Anaesth. 1997;78(2):228-9. Epub 1997/02/01.

[21] Hobson CE, Singhania G, Bihorac A. Acute kidney injury in the surgical patient. Crit Care Clin. 2015.

[22] Huen SC, Parikh CR. Predicting acute kidney injury after cardiac surgery: a systematic review. Ann Thorac Surg. 2012;93(1):337-47. S0003-4975(11)02155-2 [pii] pmid:22186469

[23] Miyagawa T, Nishida N, Ohashi J, Kimura R, Fujimoto A, Kawashima M, et al. Appropriate data cleaning methods for genome-wide association study. J Hum Genet. 2008;53(10):886-93. Epub 2008/08/13. pmid:18695938

[24] Osborne JW. Is data cleaning and the testing of assumptions relevant in the 21 st century? Front Psychol. 2013;4:370. Epub 2013/06/28. pmid:23805118

[25] Van den Broeck J, Cunningham SA, Eeckels R, Herbst K. Data cleaning: detecting, diagnosing, and editing data abnormalities. PLoS Med. 2005;2(10):e267. Epub 2005/09/06. 04PLME-PF-0256 [pii] pmid: 16138788

[26] Paul Thottakkara, TezcanOzrazgat-Baslanti, Bradley B. Hupf, ParisaRashidi, PanosPardalos, PetarMomcilovic, AzraBihorac, Application of Machine Learning Techniques to HighDimensional Clinical Data to Forecast Postoperative Complications, https://journals.plos.org/plosone/article?id=10.1371/journal.pone.0155705

[27] https://pubmed.ncbi.nlm.nih.gov/29323142/ 